\title{
Predictors and survival of primary clear cell carcinoma of liver: a population-based study of an uncommon primary liver tumor
}

\author{
Jie Wen $^{1 \#}$, Xueyan Yao ${ }^{2 \#}$, Lixiang Xue ${ }^{1}$, Abudureyimujiang Aili" ${ }^{1 \#}$, Junjie Wang ${ }^{1}$ \\ ${ }^{1}$ Department of Radiation Oncology, Peking University Third Hospital, Beijing, China; ${ }^{2}$ Department of Dermatology, Peking University People's \\ Hospital, Beijing, China \\ Contributions: (I) Conception and design: J Wang; (II) Administrative support: L Xue, J Wang; (III) Provision of study materials or patients: J Wen, \\ X Yao, A Aili; (IV) Collection and assembly of data: J Wen, X Yao, A Aili; (V) Data analysis and interpretation: J Wen, X Yao, A Aili; (VI) Manuscript \\ writing: All authors; (VII) Final approval of manuscript: All authors. \\ \#These authors contributed equally to this work. \\ Correspondence to: Junjie Wang; Abudureyimujiang Aili. Department of Radiation Oncology, Peking University Third Hospital, Beijing, China. \\ Email: junjiewang_edu@sina.cn; abudu117@126.com.
}

Background: The clinicopathological features and prognostic factors of primary clear cell carcinoma of the liver (PCCCL) remain unknown for the rarity. We aimed to determine the clinical and therapeutic characteristics of PCCCL and the effects of these factors on the prognosis.

Methods: Patients were selected from the Surveillance, Epidemiology, and End Results (SEER) database. Data were analyzed with the Kaplan-Meier, Cox proportional hazards regression analyses and the multivariable competing risk model.

Results: We included 223 PCCCL patients and the majority of them had an age under 75 years $(82.5 \%)$. Most patients were white people (63.2\%). The patients diagnosed at localized stage (63.7\%), T1 (49.8\%), N0 (96.0\%), M0 (87.4\%) and American Joint Committee on Cancer (AJCC) I (44.4\%) made up the majority of the population. More PCCCL tumors had a size beneath $4 \mathrm{~cm}(74.9 \%)$ and no vascular invasion (63.2\%). The 3-, 5-, and 10-year overall survival (OS) probabilities and disease-specific survival (DSS) rates were $35.8 \%, 24.3 \%, 14.4 \%$, and $41.6 \%, 29.4 \%, 22.2 \%$, respectively. The patients with tumor $\geq 1 \mathrm{~cm}$ [OS, hazard ratio $(\mathrm{HR})=1.822$; DSS, HR $=1.959$ ] had a higher risk of death than those patients with tumor $<1 \mathrm{~cm}$. Among surgical means, hepatectomy (OS, HR =0.070; DSS, HR =0.050) and total hepatectomy plus transplant $(\mathrm{OS}, \mathrm{HR}=0.074$; DSS, HR $=0.065$ ) were more beneficial to PCCCL.

Conclusions: PCCCL patients were inclined to be young, white people-prevalent, localized and early. PCCCL tend to had a slow growth and be weakly aggressive. However, comparing with previous reports, we found that PCCCL had a relatively poor outcome. Tumor size and surgery were the independent prognostic factors for OS and DSS.

Keywords: Primary clear cell carcinoma of the liver (PCCCL); Surveillance, Epidemiology, and End Results (SEER); pathology; demographic; prognosis

Submitted Jan 02, 2021. Accepted for publication May 28, 2021.

doi: 10.21037/tcr-21-9

View this article at: https://dx.doi.org/10.21037/tcr-21-9

\section{Introduction}

Liver cancer is the third most common cause of cancerrelated mortality, and $>90 \%$ of the cases are hepatocellular carcinoma (HCC) (1). HCC could be subdivided into clear cell, spindle cell, fibrolamellar, scirrhous, and pleomorphic types, and combined HCC with cholangiocarcinoma (2). The primary clear cell carcinoma of the liver (PCCCL) is a specific and rare histological subtype of HCC accounting for $7.5-12.5 \%$ of all liver cancers (3). PCCCL cells do not stain with hematoxylin and eosin due to the prominent cytoplasmic 
accumulation of lipid droplets and glycogens (4). Decreased number and size of organelles, and metabolic impairment can be observed in these cells (5). HCC with $>50 \%$ clear cells is generally diagnosed as PCCCL (6).

The clinical and pathological characteristics of PCCCL have been controversial for long. It is generally accepted that PCCCL has a favorable clinical outcome $(4,7,8)$, except for several reports $(9,10)$. In fact, PCCCL prognosis and prognostic factors largely remain unknown due to the limited number of cases. Additionally, studies have suggested that PCCCL mainly occurs in patients $>50$ years old (11) and was more conducive to capsule formation (12) and cirrhosis development (13). It tends to have less vascular infiltration (14) and low-grade malignancy (15). However, due to the lack of effective large case studies, the clinical and pathological features remain unclear.

As a rare HCC variant, PCCCL has largely been described in case reports or by small, single-institution studies, rendering the conclusions questionable. Hence, in this study, we expanded the number of the study cases by utilizing the Surveillance, Epidemiology, and End Results (SEER) database to clarify the tumor features, epidemiology, therapies, and prognostic factors.

We present the following article in accordance with the STROBE reporting checklist (available at https://dx.doi. org/10.21037/tcr-21-9).

\section{Methods}

The study was conducted in accordance with the Declaration of Helsinki (as revised in 2013).

\section{Study population}

The SEER database was supported by the National Cancer Institute and contained the information of 18 population based registered cancer institutes. The US population accounts for $28.0 \%$ of the SEER database. SEER program was established to collect cancer patient's information including cancer incidence, prevalence, treatments, clinicopathological features and survival data from U.S. cancer registries, which is broadly represents the population of the US population. It serves to minimize the potential biases, reduce the cancer burden and provide first-hand evidence for clinicians to perform studies focusing on oncologic disease (16). As the patients data in the SEER dataset are publicly available, therefore, no approval was required from any institutional review board (17).
The study we designed was a population based longitudinal cohort study. And we utilized the SEER database to identify patients diagnosed with PCCCL (SEER codes 8174/3) from 2004 to 2016 according to the 3rd edition of International Classification of Disease for Oncology (ICD-0-3) and the 6th edition of American Joint Committee on Cancer (AJCC). The exclusion criteria were as follows: (I) diagnostic confirmation that is not "positive histology"; (II) sequence number that is not "one primary only"; (III) AJCC T that is "TX, T0, NA, or blank"; (IV) AJCC N that is "NX, NA, or blank"; (V) AJCC M that is "MX, NA, or blank"; (VI) reason no cancer-directed surgery that is "recommended, unknown if performed" and "unknown; death certificate; or autopsy only"; (VII) race recode that is "unknown"; (VIII) size recode that is "unknown".

The patient information including diagnostic confirmation, histology/behavior (ICD-O-3), SEER combined summary stage, AJCC stage (6th), AJCC T stage (6th), AJCC M stage (6th), AJCC N stage (6th), surgery primary site $(1998+)$, radiation therapy, tumor size, CS extension, CS lymph nodes [2004-2015], CS metastasis at distance [2004-2015], SEER cause-specific death classification, survival months, sequence number, age at diagnosis, gender, race, and pathological grade were extracted from the database. The details of chemotherapy was not included in the SEER database. No private identity information were acquired from the SEER database.

\section{Definition of variables and grouping criteria}

Age was defined as the age at diagnosis as PCCCL and was classified into 2 groups: " $<75$ " and " $\geq 75$ " years old. Race was recoded as follows: white, black, or others. Sex was described as male and female. Tumor size was assigned into three groups: " $<1 \mathrm{~cm}$ " and " $\geq 1 \mathrm{~cm}$ ". AJCC $T$ stage was classified as four subgroups: "T1", "T2", "T3" and "T4". AJCC N stage was categorized into two subgroups: "N0" and "N1". AJCC M stage was divided into two subgroups: "MO" and "M1". AJCC stage was assigned into four groups: "I", "II", "III (IIIA, IIIB, IIIC)", and "IV". SEER stage was classified as three subgroups: "localized", "regional" and "distant". The radiotherapy was divided into two groups: "Yes" which means the patients underwent radiotherapy and "No" means not. The methods of surgery was assigned into the following six types: "none", "local tumor destruction", "lobectomy", "extended lobectomy", "wedge or/and segmental resection", "hepatectomy" and "total hepatectomy plus transplant" 


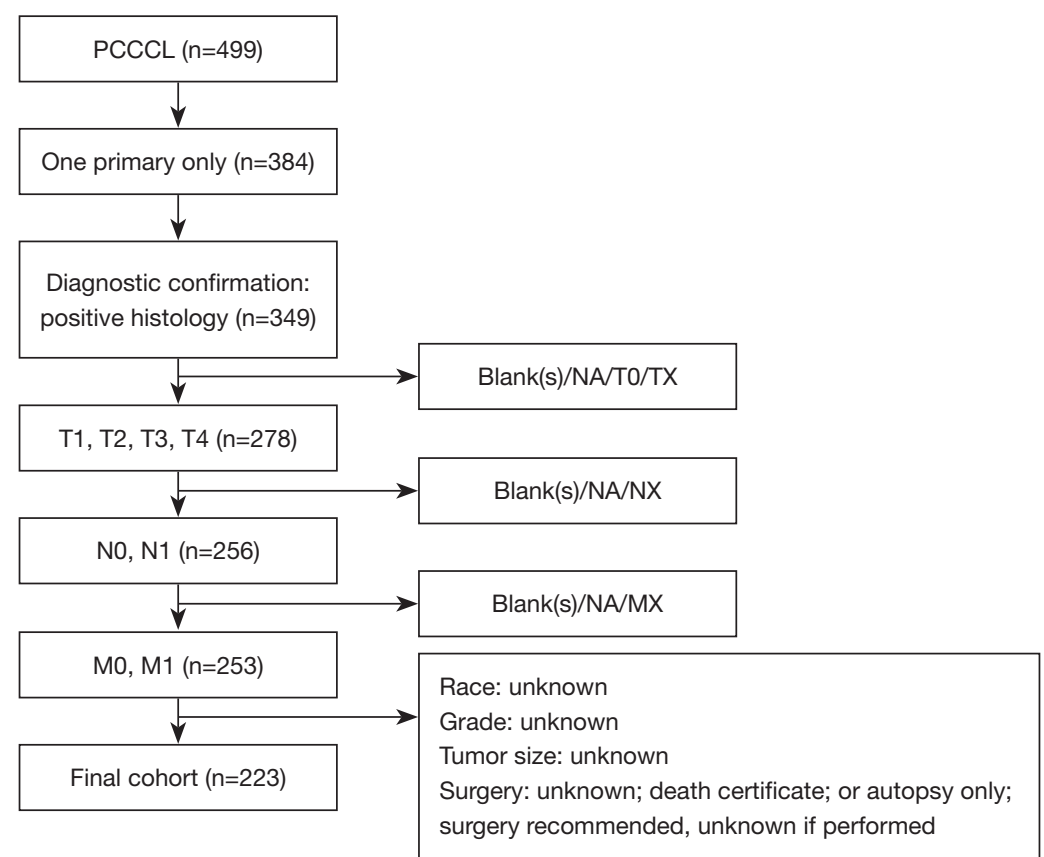

Figure 1 Flow diagram of study cohort selection. A flow diagram describing the inclusion and exclusion criteria for the patient selection. According to the 6th edition of AJCC, we excluded those patients with T0, TX, NX, MX and unspecific information, including unknown surgery methods, unknown race and unknown tumor size. Those PCCCL patients with blank information and NA were also excluded from the cohort. AJCC, American Joint Committee on Cancer; PCCCL, primary clear cell carcinoma of liver; NA, not applicable.

and "partial hepatectomy plus bile duct excision". Tumor extension was categorized as follows: "no vascular invasion", "intrahepatic vascular invasion", "gallbladder extension", "intrahepatic vascular invasion plus gallbladder extension", "surface of parenchyma extension", "major vascular invasion: major branch(es) of portal or hepatic vein(s)", and "hepatic artery or vena cava invasion", "coronary, falciform, hepatoduodenal, hepatogastric, round (of liver), triangular, perforation of visceral peritoneum, parietal peritoneum, pancreas, pleura, stomach extension".

\section{Statistical analysis}

The demographic, clinical, and pathological characteristics of PCCCL patients were analyzed with basic statistics. The primary endpoints were overall survival (OS) and disease-specific survival (DSS). OS and DSS were defined as the time intervals from diagnosis to death by any cause and PCCCL-related reasons, respectively. The KaplanMeier method and log-rank test were used to validate the prognostic factors and statistical significance for the OS and DSS. The univariate and multivariable Cox proportional hazards regression were performed to identify the independent prognostic variables. The hazard ratio (HR) and 95\% confidence interval (CI) were measured for assessing the correlation strength between each variable and survival. The multivariable competing risk model (Fine \& Gray model) was used to validate the independent factors affecting the risk for DSS. The GraphPad Prism 6 software was applied to draw the figures and charts. SPSS 19.0 (SPSS, Inc., Chicago, IL, USA) was for data analysis. SEER*STAT version 8.3.5 (Surveillance Research Program, NCI, Bethesda, MD, USA) was utilized to extract patient information from the SEER database (18). And the StataMP 14 was utilized to make competing risk model analysis. $\mathrm{P}<0.05$ was considered statistically significant.

\section{Results}

\section{Patient population}

PCCCL cases during 2004-2016 ( $\mathrm{n}=499)$ were extracted from the SEER database. Subsequently, 223 cases were recruited to our study cohort according to the inclusion and exclusion criteria as shown in Figure 1. Patient-related general information was listed in Table 1 . The average age 
Table 1 Characteristics of patients with PCCCL diagnosed from 2004 to 2016 obtained from the SEER database

\begin{tabular}{|c|c|c|}
\hline Parameter & Subgroup & Number (\%) \\
\hline \multirow[t]{2}{*}{ Age } & $<75$ & $184(82.5)$ \\
\hline & $\geq 75$ & $39(17.5)$ \\
\hline \multirow[t]{2}{*}{ Race } & White & $141(63.2)$ \\
\hline & Others & $55(24.7)$ \\
\hline \multirow[t]{2}{*}{ Sex } & Male & $128(57.4)$ \\
\hline & Female & $95(42.6)$ \\
\hline Summary stage & Localized & $142(63.7)$ \\
\hline \multirow[t]{4}{*}{ T stage } & $\mathrm{T} 1$ & $111(49.8)$ \\
\hline & $\mathrm{T} 2$ & $46(20.6)$ \\
\hline & T3 & $55(24.7)$ \\
\hline & $\mathrm{T} 4$ & $11(4.9)$ \\
\hline \multirow[t]{2}{*}{$\mathrm{N}$ stage } & NO & $214(96.0)$ \\
\hline & $\mathrm{N} 1$ & $9(4.0)$ \\
\hline \multirow[t]{2}{*}{ M stage } & MO & $195(87.4)$ \\
\hline & M1 & $28(12.6)$ \\
\hline \multirow{3}{*}{ AJCC stage } & ॥ & $42(18.8)$ \\
\hline & III & $54(24.2)$ \\
\hline & IV & $28(12.6)$ \\
\hline \multirow[t]{8}{*}{ Surgery } & None & $107(48.0)$ \\
\hline & Local tumor destruction & $21(9.4)$ \\
\hline & Lobectomy & $28(12.6)$ \\
\hline & Extended lobectomy & $4(1.8)$ \\
\hline & Wedge or/and segmental resection & $47(21.1)$ \\
\hline & Hepatectomy & $4(1.8)$ \\
\hline & Total hepatectomy + transplant & $11(4.9)$ \\
\hline & Partial hepatectomy + bile duct excision & $1(0.4)$ \\
\hline
\end{tabular}

Table 1 (continued) 
Table 1 (continued)

\begin{tabular}{lll}
\hline Parameter & Subgroup & Number (\%) \\
\hline Extension & No vascular invasion & 141 (63.2) \\
& Intrahepatic vascular invasion and/or gallbladder extension & $26(11.7)$ \\
& Surface of parenchyma extension & 29(13.0) \\
& Major vascular invasion: major branch(es) of portal or hepatic vein(s) \\
& Hepatic artery or vena cava invasion & 16 (7.2) \\
& Coronary, falciform, hepatoduodenal, hepatogastric, round (of liver), triangular, perforation of & 9 (4.0) \\
& visceral peritoneum, parietal peritoneum, pancreas, pleura, stomach extension
\end{tabular}

Table 1: characteristics of patients with PCCCL. PCCCL, primary clear cell carcinoma of liver; SEER, Surveillance, Epidemiology, and End Results.

at diagnosis was $63.8 \pm 12.4$ years with $57.4 \%$ male; $63.2 \%$, $12.1 \%$, and $24.7 \%$ were white, black, and of other ethnicity, respectively. Tumor was present in $74.9 \%(<1 \mathrm{~cm})$ and $25.1 \%(\geq 1 \mathrm{~cm})$ of the patients. Nearly half the patients (49.8\%) were diagnosed at T1, 20.6\% at T2, 24.7\% at $\mathrm{T} 3$, and $4.9 \%$ at $\mathrm{T} 4$ stages. The overwhelming majority of the patients (N0,96.0\%) had no regional lymph node or tumor distant metastasis (M0, 87.4\%). The patients diagnosed as early AJCC stage I, or stages II, III, and IV accounted for $44.4 \%, 18.8 \%, 24.2 \%$, and $12.6 \%$ of the sample size, respectively. Regarding SEER staging, 63.7\%, $22.8 \%$, and $13.5 \%$ of the patients had localized, regional, and distant extent of PCCCL, respectively. The patients with no vascular invasion corresponded to $63.2 \%$. Only a few people $(2.7 \%)$ underwent radiotherapy. Approximately half the patients (48.0\%) had no surgical treatment; $9.4 \%$ and $12.6 \%$ received local tumor destruction and lobectomy, respectively; $1.8 \%$ underwent extended lobectomy; $21.1 \%$, $1.8 \%, 4.9 \%$ and $0.4 \%$ underwent wedge or/and segmental resection, hepatectomy, total hepatectomy plus transplant and partial hepatectomy plus bile duct excision, respectively.

\section{Clinical prognosis and survival analysis}

To understand whether these variables would affect the survival of patients, we first analyzed the association strength between each variable and survival. The Kaplan-Meier survival analysis showed that the 5-year OS and DSS rates were $24.3 \%$ and $29.4 \%$, respectively, and thus indicated a relatively poor clinical (Figure $2 A, B$ ). The median OS and DSS were 21 and 26 months, respectively (Table 2). The Kaplan-Meier analysis results showed that the patients $\geq 75$ years old exhibited a poorer outcome than that of the younger patients, with a median OS of 15 vs. 25 months (logrank $\mathrm{P}=0.048)$. However, age had no statistically significant effect on the DSS (log-rank $\mathrm{P}=0.230$ ) (Figure $3 A, B$, Table 2). Additionally, higher $T$ stage correlated with decreased $O S$ and DSS (log-ranks $\mathrm{P}<0.001$ for both) with patients at $\mathrm{T} 4$ or T3 stage having a dismal prognosis (T4 median OS and DSS, both 9 months; T3 median OS and DSS, both 12 months) (Figure 3C,D, Table 2). Poor prognosis could be observed in the patients with $\mathrm{N} 1$ stage (OS log-rank $\mathrm{P}=0.017$, DSS logrank $\mathrm{P}=0.004$, median OS and DSS, both 8 months) and M1 stage (OS log-rank $\mathrm{P}<0.001$, median OS: 7 months; DSS log-rank $\mathrm{P}<0.001$, median DSS: 8 months) (Figure $3 E, F, G, H$, Table 2). Regarding AJCC stage, shorter survival was observed in the stage IV patients (median OS and DSS: 7 and 8 months, respectively) and stage III patients (median OS and DSS: both 13 months), while, the opposite trend could be observed in those with stage II (median OS: 26 months; median DSS: 30 months) and stage I (median OS: 41 months; median DSS: 50 months) (OS and DSS logranks, both $\mathrm{P}<0.001$ ) (Figure $4 A, B$, Table 2). Additionally, later summary stage (distant) correlated with decreased survival (median OS: 6 months; median OS: 7 months) compared with localized stage (median OS and DSS: 34 and 42 months, respectively) and regional stage (median OS and DSS, both 13 months) (OS and DSS log-ranks, both $\mathrm{P}<0.001$ ) (Figure $4 C, D$, Table 2). The median OS and DSS of the patients with tumor $<1 \mathrm{~cm}$ were 30 and 36 months, respectively, which were longer than those of the patients with tumors $\geq 1 \mathrm{~cm}$ (median OS and DSS, both 12 months) (OS and DSS log-ranks, both $\mathrm{P}<0.001$ ) (Figure 4E,F, Table 2). Shorter survival could be observed in patients with parenchyma surface extension (median OS and DSS, both 11 months), major vascular invasion (median OS 

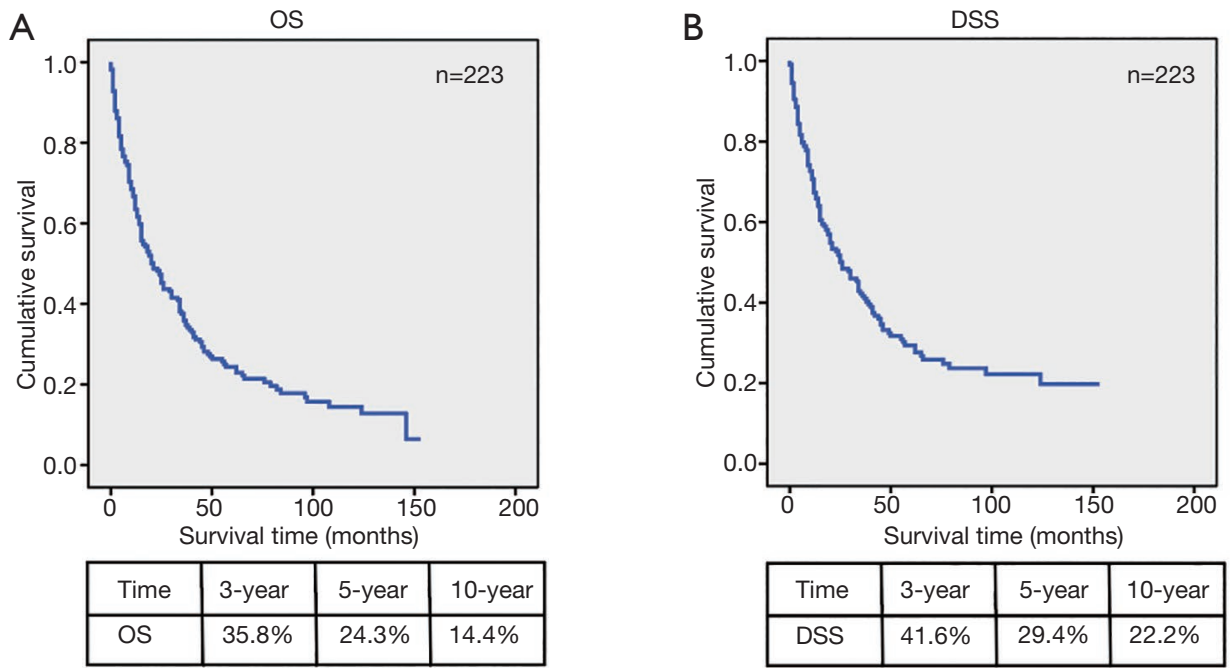

Figure 2 Kaplan-Meier analysis: OS curves (A) and DSS curves (B) of PCCCL for the entire cohort. Three-, 5-, and 10-year OS rates were $35.8 \%, 24.3 \%$, and 14.4\%, respectively. Three-, 5-, and 10-year DSS rates were 41.6\%, 29.4\%, and 22.2\%, respectively. Median OS and DSS were 21 and 26 months, respectively. OS, overall survival; DSS, disease-specific survival; PCCCL, primary clear cell carcinoma of liver.

and DSS: both 12 months), hepatic artery or vena cava invasion (median OS and DSS, both 4 months), or coronary, falciform, hepatoduodenal, hepatogastric, round (of liver), triangular, perforation of visceral peritoneum, parietal peritoneum, pancreas, pleura, stomach extension (median OS and DSS: 13 and 3 months, respectively) compared with those without vascular invasion (median OS and DSS: 34 and 42 months, respectively) (OS and DSS log-ranks, both $\mathrm{P}<0.001$ ) (Figure $5 A, B$, Table 2). With reference to different surgery methods, a median OS and DSS of 37 and 42 months, respectively, were observed for patients receiving local tumor destruction, 56 (OS) and 62 months (DSS) for patients undergoing wedge or/and segmental resection, 34 months (for both OS and DSS) for patients accepting lobectomy, 41 months (for both OS and DSS) for those having extended lobectomy, 62 months (for both OS and DSS) for those with hepatectomy, and 124 months (OS) for those after total hepatectomy plus transplant. Conversely, the clinical outcome of the patients without any surgery was poor (median OS and DSS: 9 and 10 months, respectively; OS and DSS log-ranks, both $\mathrm{P}<0.001$ ) (Figure 5C,D, Table 2). However, no statistically significant differences in OS and DSS could be observed in patients of both genders (OS log-rank $\mathrm{P}=0.756$, DSS log-rank $\mathrm{P}=0.811$ ) and different races. (OS log-rank $\mathrm{P}=0.394$, DSS log-rank $\mathrm{P}=0.869$ ) (Figure $6 A, B, C, D$ ). It should be noted that there was no significant difference in OS (logrank, $\mathrm{P}=0.960$ ) or DSS (log-rank, $\mathrm{P}=0.785$ ) in patients with or without radiation (Figure 6E,F, Table 2).

\section{Uni- and multivariate analyses}

To further dissect the independent predictors for OS and DSS of the PCCCL patients, we performed uni- and multivariate analyses. Summary stages, surgery, tumor size, TNM stage, AJCC, and extension were markedly correlated with both the OS and DSS (Table 2). The univariate analysis showed that the summary stage was a significant predictor of OS and DSS ( $\mathrm{P}<0.001$ for both). Relative to patients with localized stage, increased HR could be observed in the patients with distant stage (OS: HR $=3.545,95 \%$ CI: 2.288 5.492; DSS: HR $=3.718,95 \%$ CI: 2.313-5.977) or regional stage (OS: HR $=1.931,95 \%$ CI: $1.347-2.769$; DSS: HR $=2.176,95 \%$ CI: $1.482-3.194)$. Lower survival occurred in M1 stage (OS: HR =2.771, 95\% CI: 1.800-4.266; DSS: HR $=2.738,95 \%$ CI: $1.715-4.372)$, N1 stage (OS: HR $=2.305$, 95\% CI: 1.121-4.738; DSS: HR $=2.719,95 \%$ CI: $1.317-$ 5.616), and T3 (OS: HR =2.676, 95\% CI: 1.854-3.863; DSS: $\mathrm{HR}=3.511,95 \% \mathrm{CI}: 2.370-5.203)$ and T4 stages (OS: HR $=2.562,95 \%$ CI: $1.317-4.981$; DSS: HR $=3.452$, 95\% CI: 1.751-6.802) than in M0, N0, and T1 stages, respectively. The patients with tumors $<1 \mathrm{~cm}$ had poorer prognosis (OS: HR $=1.806,95 \%$ CI: 1.284-2.538; DSS: $\mathrm{HR}=1.992,95 \%$ CI: $1.388-2.859)$ than those with tumors $\geq 1 \mathrm{~cm}$. PCCCL extended in parenchyma surface (OS: HR $=2.145,95 \%$ CI: $1.383-3.327$; DSS: HR $=2.595,95 \%$ CI: 
Table 2 Results of univariate analysis of potential patient characteristics influencing DSS and OS using the Cox proportional HRs

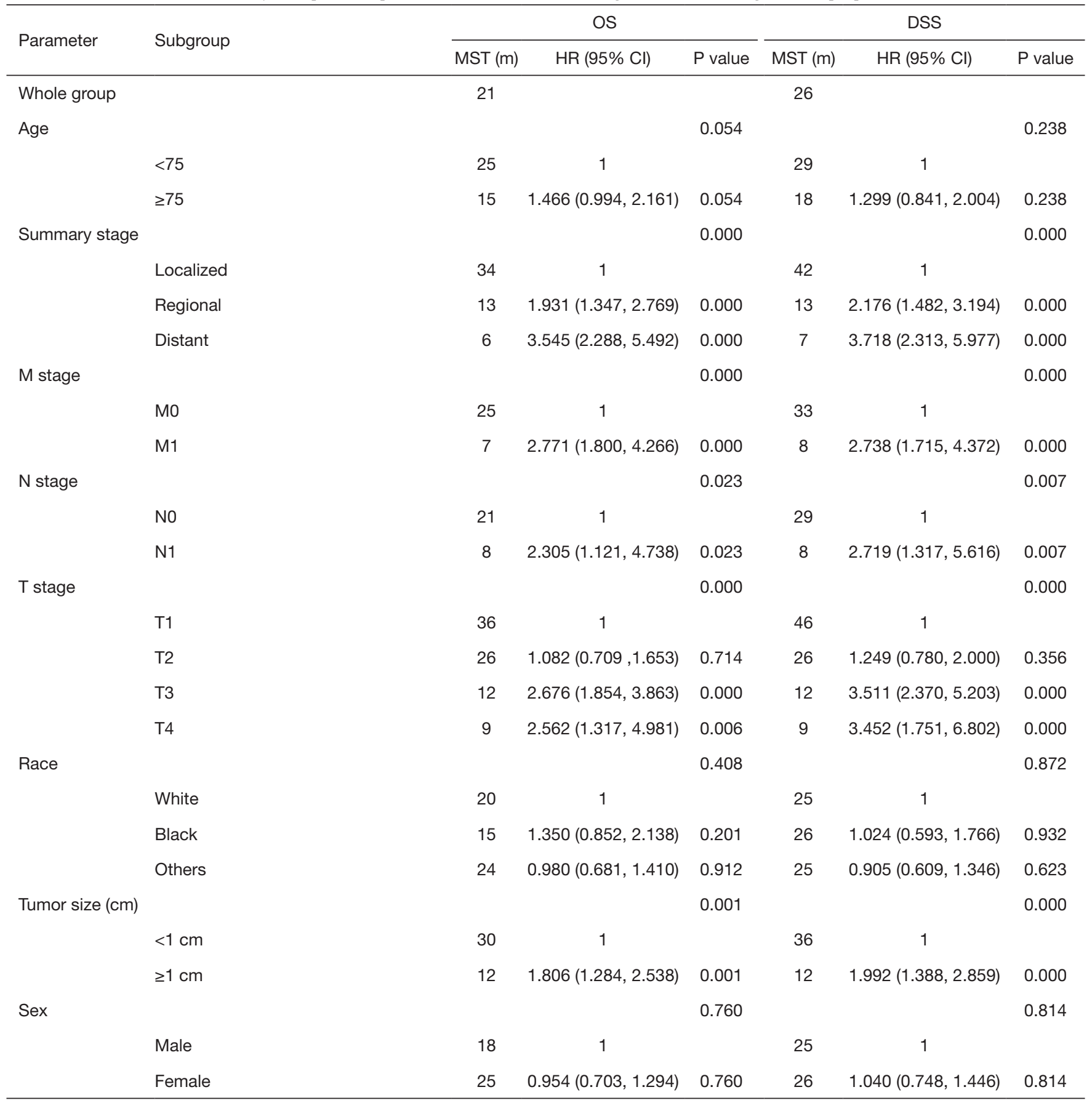

Table 2 (continued) 
Table 2 (continued)

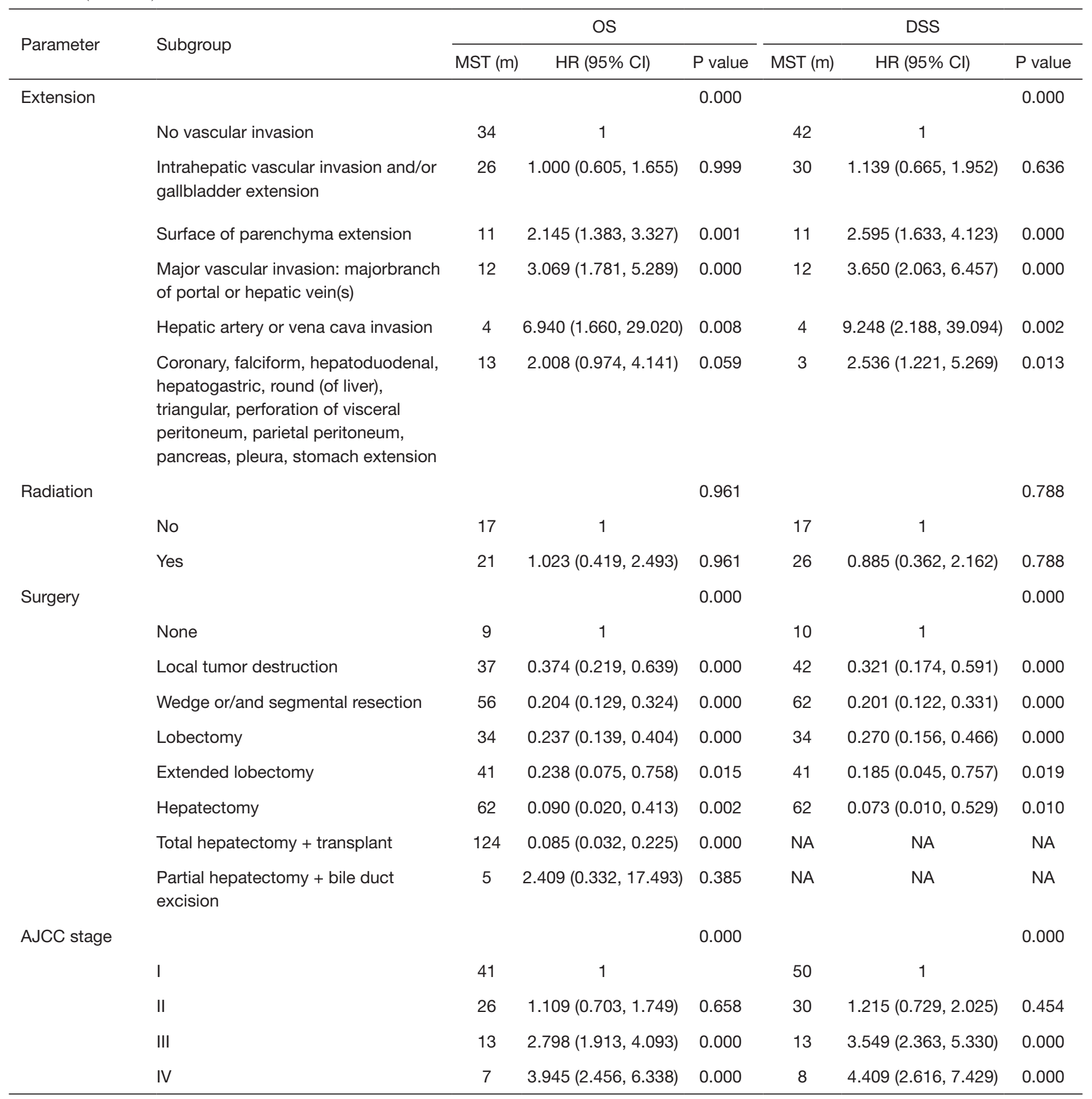

Table 2: SEER summary stage, M stage, N stage, T stage, tumor size $(\mathrm{cm})$, extension status, surgery and AJCC stage were significantly related to the OS and DSS of PCCCL patients. 0.000 means $\mathrm{P}<0.001$. DSS, disease-specific survival; OS, overall survival; HR, hazard ratio; MST, median survival time; Cl, confidence interval; NA, not applicable; AJCC, American Joint Committee on Cancer; SEER, Surveillance, Epidemiology, and End Results; PCCCL, primary clear cell carcinoma of liver. 
A

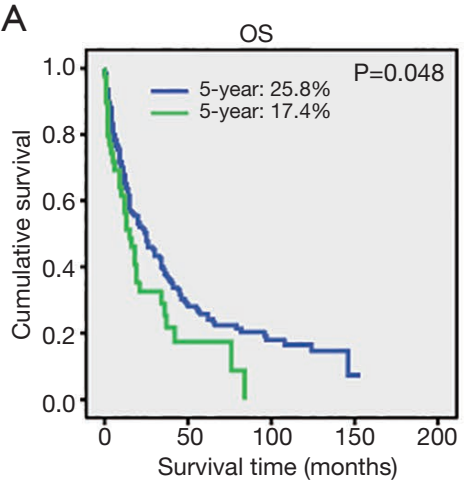

C

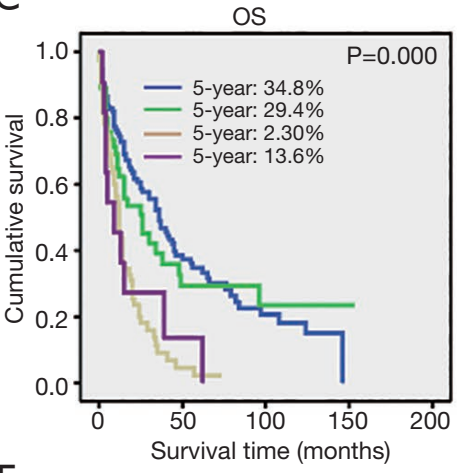

E

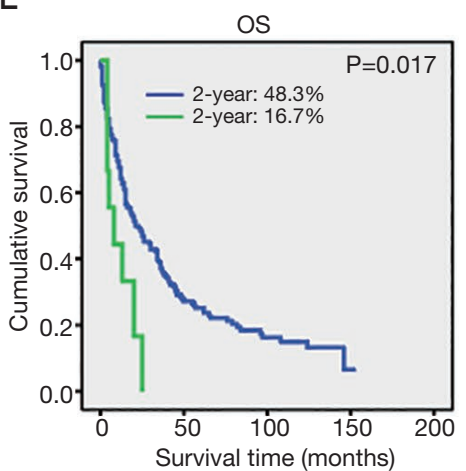

G

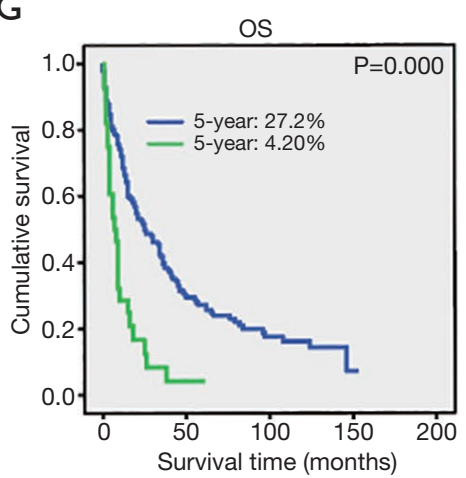

B

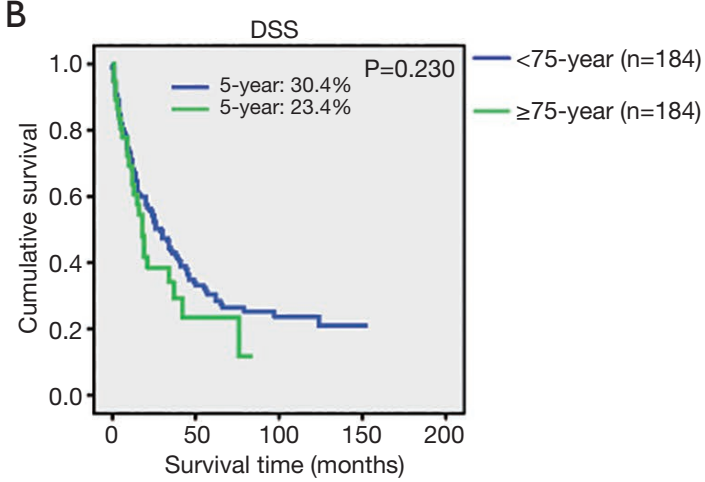

D

DSS

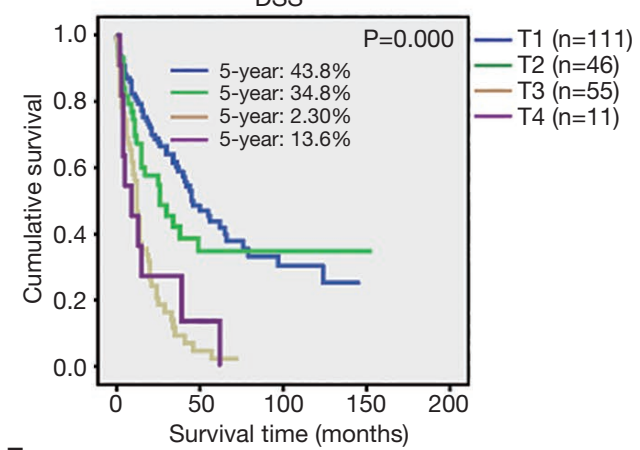

$\mathrm{F}$

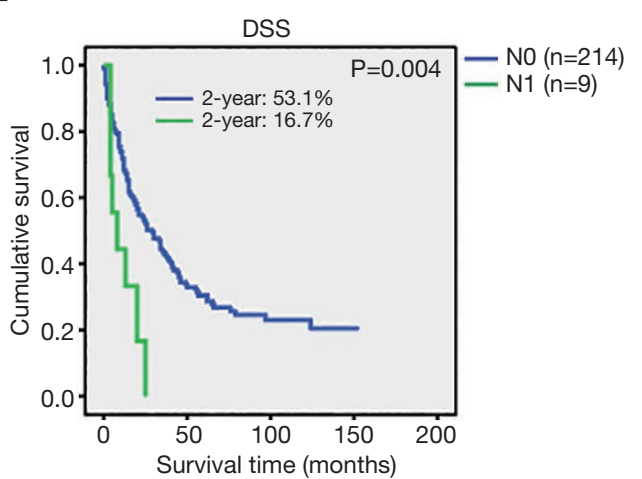

$\mathrm{H}$

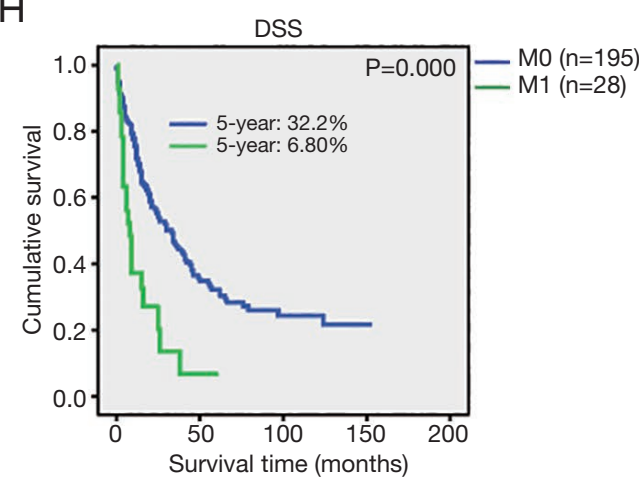

Figure 3 Kaplan-Meier analysis of the variables. Kaplan-Meier estimates of the OS and DSS stratified by (A,B) age, (C,D) AJCC T stage, (E,F) AJCC N stage, (G,H) AJCC M stage. And the 2- or 5-year survival rate of PCCCL patients in each group were listed in the corresponding pictures. 0.000 means $\mathrm{P}<0.001$. OS, overall survival; DSS, disease-specific survival; AJCC, American Joint Committee on Cancer; PCCCL, primary clear cell carcinoma of liver. 
A

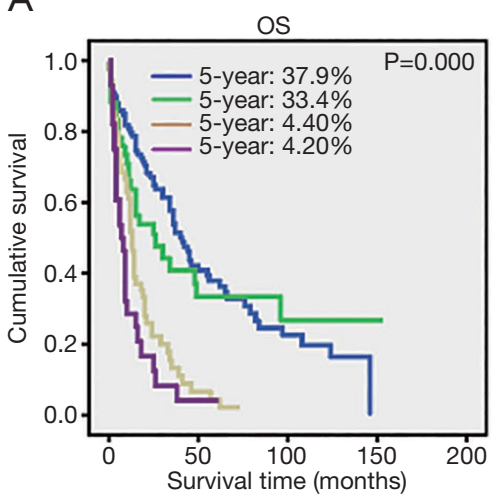

C

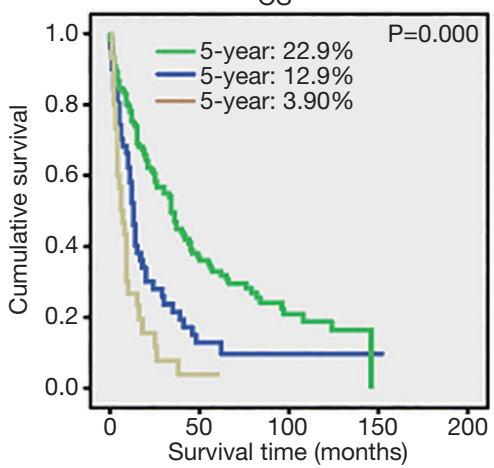

E

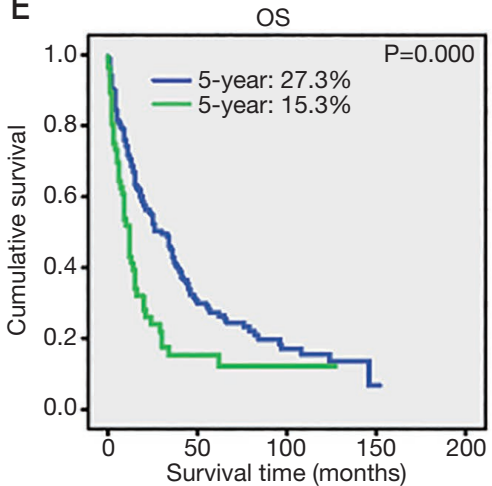

B

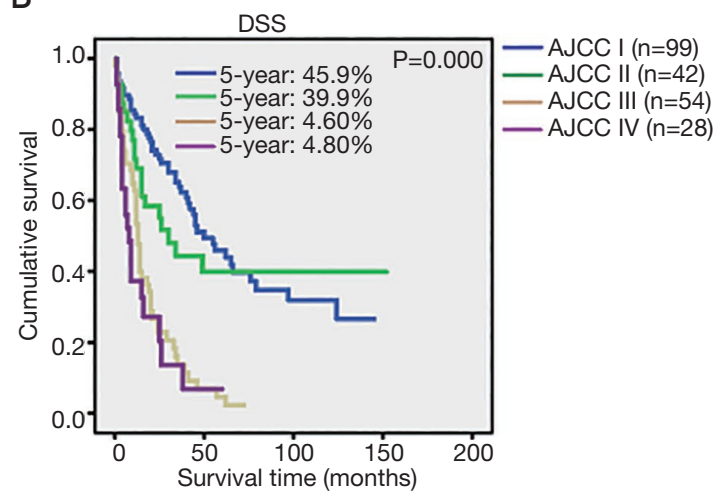

$\mathrm{D}$

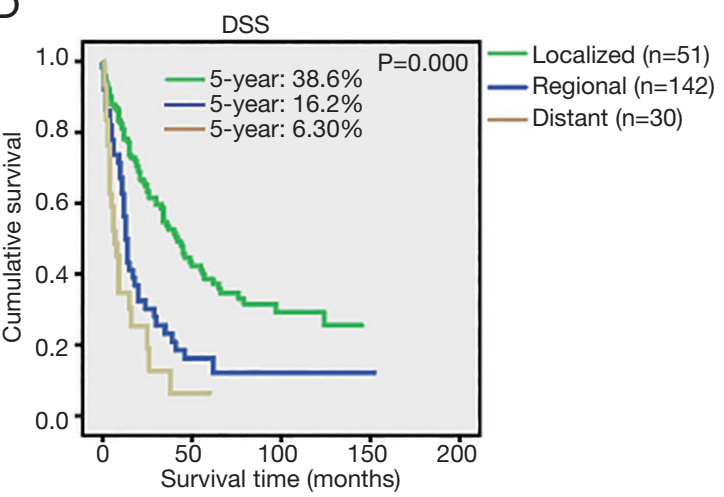

$\mathrm{F}$

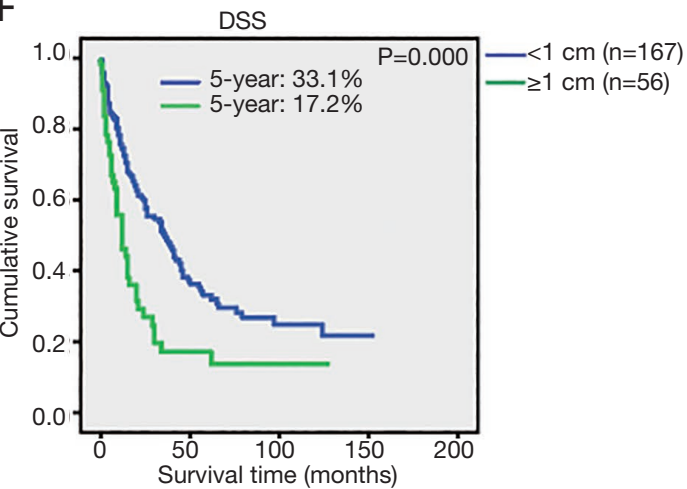

Figure 4 Kaplan-Meier analysis of the variables (continued from Figure 3). Kaplan-Meier estimates of the OS and DSS stratified by $(A, B)$ AJCC stage, (C,D) summary stage, (E,F) tumor size. And the 5-year survival rate of PCCCL patients in each group were listed in the corresponding pictures. 0.000 means $\mathrm{P}<0.001$. OS, overall survival; DSS, disease-specific survival; AJCC, American Joint Committee on Cancer; PCCCL, primary clear cell carcinoma of liver.

1.633-4.123), major branches of portal or hepatic vein(s) (OS: HR $=3.069,95 \%$ CI: $1.781-5.289$; DSS: HR =3.650, 95\% CI: 2.063-6.457), hepatic artery or vena cava (OS: HR $=6.940,95 \%$ CI: 1.660-29.020; DSS: HR $=9.248,95 \%$ CI: 2.188-39.094), or with coronary, falciform, hepatoduodenal, hepatogastric, round (of liver), triangular, perforation of visceral peritoneum, parietal peritoneum, pancreas, pleura, or stomach extension (OS: HR $=2.008,95 \%$ CI: $0.974-$ 4.141; DSS: HR =2.536, 95\% CI: $1.221-5.269)$ had higher risks than those without vascular invasion. Compared with patients without surgery, those who underwent local tumor destruction (OS: HR $=0.374,95 \%$ CI: 0.219-0.639; DSS: $\mathrm{HR}=0.321,95 \%$ CI: $0.174-0.591)$, wedge or/and segmental resection (OS: HR $=0.204$, 95\% CI: 0.129-0.324; DSS: 

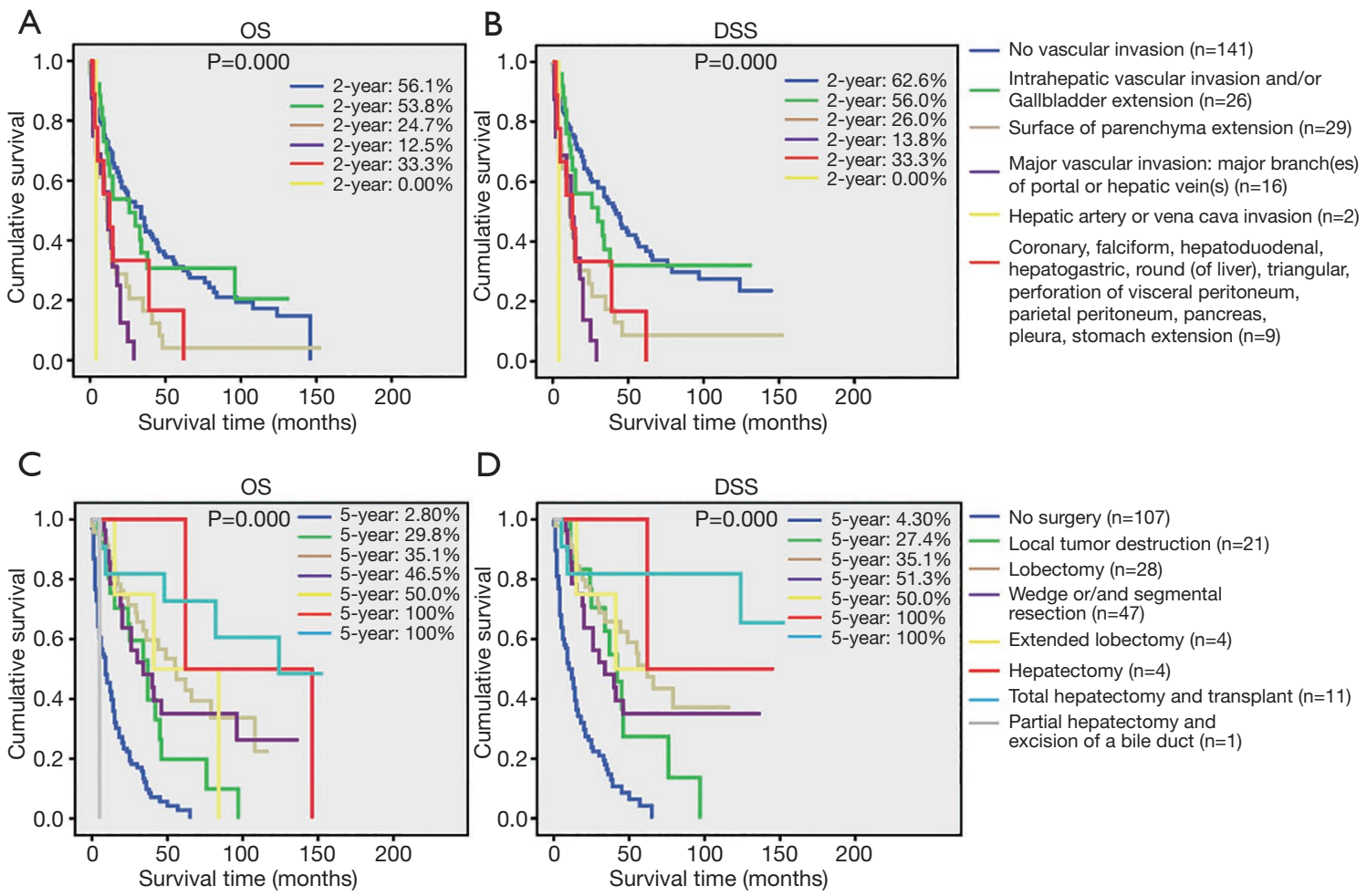

Figure 5 Kaplan-Meier analysis of the variables (continued from Figure 4). Kaplan-Meier estimates of the OS and DSS stratified by (A,B) extension, (C,D) surgery. And the 2- or 5-year survival rate of PCCCL patients in each group were listed in the corresponding pictures. 0.000 means $\mathrm{P}<0.001$. OS, overall survival; DSS, disease-specific survival; PCCCL, primary clear cell carcinoma of liver.

HR $=0.201,95 \%$ CI: $0.122-0.331)$, lobectomy (OS: HR $=0.237,95 \%$ CI: 0.139-0.404; DSS: HR $=0.270,95 \%$ CI: 0.156-0.466), extended lobectomy (OS: HR 0.238, 95\% CI: 0.075-0.758; DSS: HR $=0.185,95 \%$ CI: $0.045-0.757$ ), hepatectomy (OS: HR $=0.090,95 \%$ CI: $0.020-0.413$; DSS: $\mathrm{HR}=0.073,95 \%$ CI: $0.010-0.529)$ or total hepatectomy plus transplant (OS: HR $=0.085$, 95\% CI: 0.032-0.225) had reduced risks of death. Patients diagnosed as AJCC III (OS: HR $=2.798$, 95\% CI: 1.913-4.093; DSS: HR $=3.549$, 95\% CI: $2.363-5.330$ ) or AJCC IV (OS: HR $=3.945$, 95\% CI: 2.456-6.338; DSS: HR =4.409, 95\% CI: 2.616-7.429) showed significantly high risks of death than those with AJCC I or II. However, age, race, sex and radiotherapy had no impacts on the hazard rate.

Next step, to determine the independent prognostic factors, we performed multivariate cox proportional analysis model of OS and DSS (Table 3) for those variables whose $\mathrm{P}$ value $<0.05$ according to the univariate analyses results in Table 2. As shown in Table 3, for the whole cohort, tumor size and surgery were independent determinants of OS and DSS. Relative to the patients with tumor $<1 \mathrm{~cm}$, those with lesion $\geq 1 \mathrm{~cm}$ possessed shorter survival (OS: $\mathrm{HR}=1.822$, 95\% CI: 1.222-2.717; DSS: HR $=1.959$, 95\% CI: $1.283-$ 2.990). Receiving surgery had improved OS and DSS. Using no surgery as a reference, patients accepting local tumor destruction (OS: HR $=0.505,95 \%$ CI: 0.285-0.895; DSS: HR $=0.464,95 \%$ CI: 0.242-0.890), wedge or/and segmental resection (OS: HR $=0.237,95 \%$ CI: 0.146-0.386; DSS: HR $=0.240,95 \%$ CI: $0.142-0.405$ ), lobectomy (OS: $\mathrm{HR}=0.216,95 \%$ CI: 0.118-0.394; DSS: HR $=0.244$, 95\% CI: 0.131-0.454), extended lobectomy (OS: HR $=0.349,95 \%$ CI: $0.107-1.138$; DSS: HR $=0.296,95 \%$ CI: $0.070-1.243$ ), hepatectomy (OS: HR $=0.070,95 \%$ CI: $0.016-0.312$; DSS: HR $=0.050,95 \%$ CI: $0.006-0.386$ ), and total hepatectomy plus transplant (OS: HR $=0.074,95 \%$ CI: $0.025-0.219$; DSS: HR $=0.065$, 95\% CI: $0.018-0.237$ ) had a good clinical outcome. In the final model as shown in Table 4, tumor size and surgery had impacts on the OS and DSS. The patients having small tumor $(<1 \mathrm{~cm})$ and undergoing surgery possessed a better clinical outcome.

Consistent with the above results, the regression results of multivariate competitive risk model for PCCCL 
A

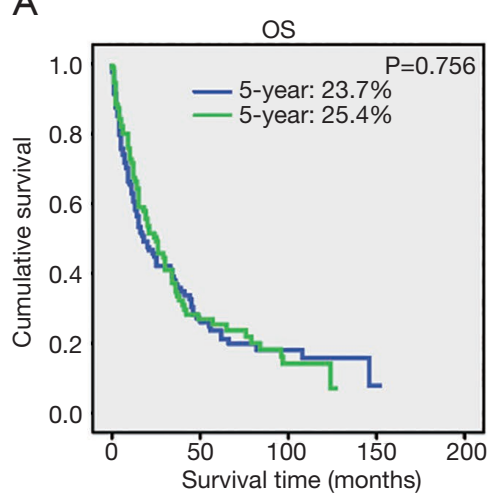

C

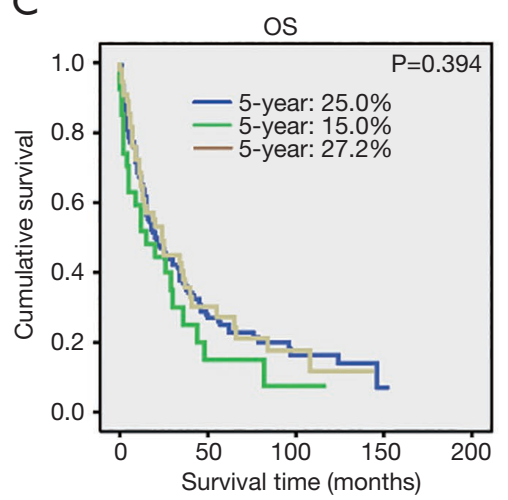

$\mathrm{E}$

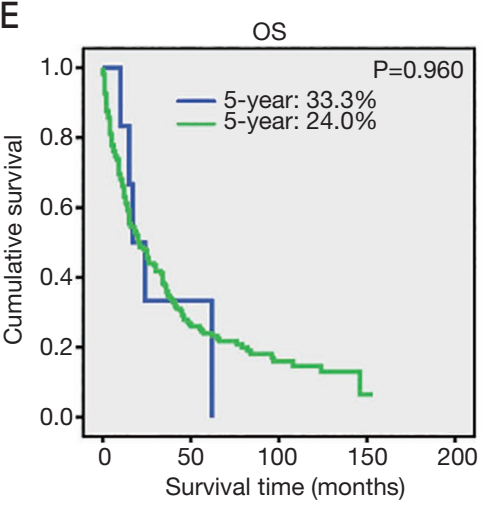

B

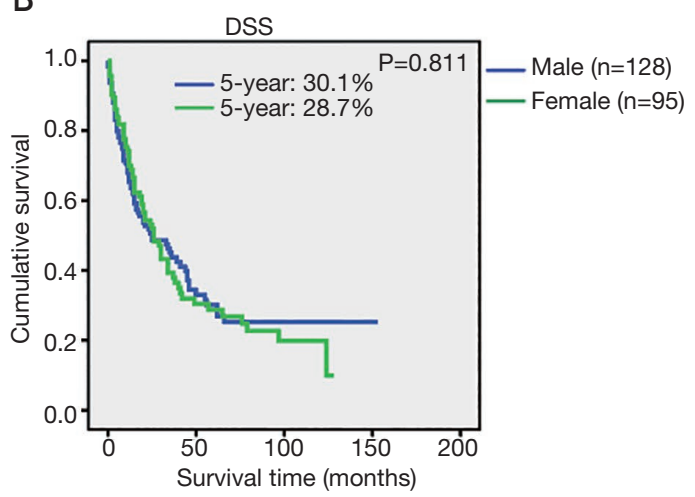

D

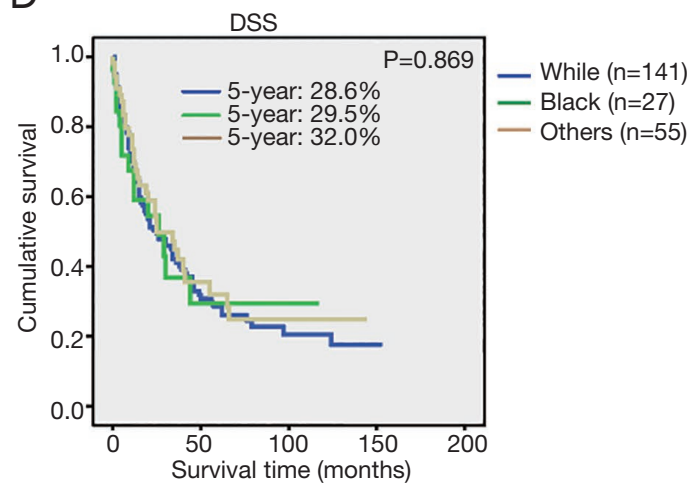

$\mathrm{F}$

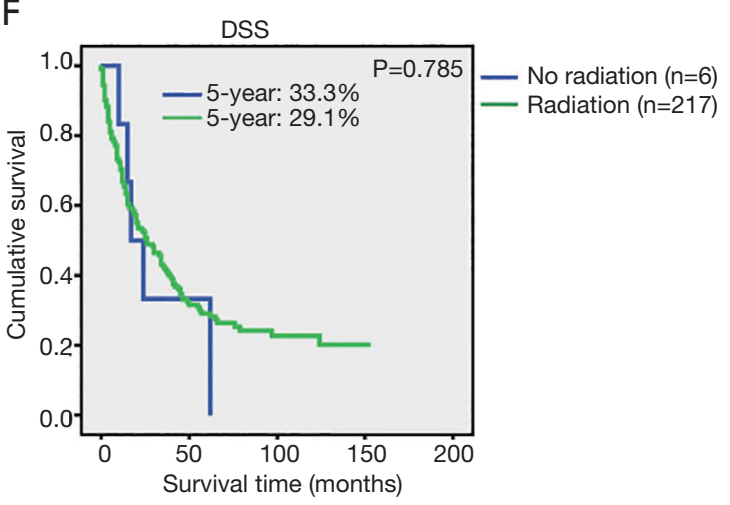

Figure 6 Kaplan-Meier analysis of the variables (continued from Figure 5). Kaplan-Meier estimates of the OS and DSS stratified by (A,B) sex, $(\mathrm{C}, \mathrm{D})$ race, $(\mathrm{E}, \mathrm{F})$ radiation. And the 5-year survival rate of PCCCL patients in each group were listed in the corresponding pictures. OS, overall survival; DSS, disease-specific survival; PCCCL, primary clear cell carcinoma of liver.

showed that tumor size and surgery were significantly correlated with DSS (Table 5), and tumor $\geq 1 \mathrm{~cm}$ had higher risk for death due to PCCCL comparing to those patients with tumor $<1 \mathrm{~cm}$. As for surgery, we found that partial hepatectomy along with bile duct excision, total hepatectomy and transplant combination, hepatectomy and extended lobectomty were more inversely associated with risk for DSS, followed by lobectomy, wedge or/and segmental resection and local destruction (Figure $7 A, B)$.

\section{Discussion}

Due to the glycogen accumulation and fat storage in the cytoplasm, cytoplasmic clearing to the hematoxylin-eosin 
Table 3 Results of multivariate analysis of potential patient characteristics influencing DSS and OS using the Cox proportional HRs

\begin{tabular}{|c|c|c|c|c|c|c|c|}
\hline Parameter & Subgroup & \multicolumn{3}{|c|}{ OS } & \multicolumn{3}{|c|}{ DSS } \\
\hline \multirow[t]{3}{*}{ Summary stage } & & & & 0.323 & & & 0.331 \\
\hline & Localized & 1 & & & 1 & & \\
\hline & Regional & 0.799 & $(0.332,1.921)$ & 0.616 & 0.835 & $(0.326,2.138)$ & 0.706 \\
\hline \multirow[t]{3}{*}{ M stage } & & & & 0.999 & & & 0.806 \\
\hline & MO & 1 & & & 1 & & \\
\hline & M1 & 0.999 & $(0.141,7.063)$ & 0.999 & 0.774 & $(0.100,5.972)$ & 0.806 \\
\hline $\mathrm{N}$ stage & & & & 0.712 & & & 0.999 \\
\hline \multirow[t]{5}{*}{ T stage } & & & & 0.692 & & & 0.866 \\
\hline & $\mathrm{T} 1$ & 1 & & & 1 & & \\
\hline & $\mathrm{T} 2$ & 0.797 & $(0.207,3.071)$ & 0.742 & 1.311 & $(0.311,5.530)$ & 0.712 \\
\hline & T3 & 0.595 & $(0.181,1.953)$ & 0.392 & 0.901 & $(0.243,3.344)$ & 0.876 \\
\hline & $\mathrm{T} 4$ & 0.856 & $(0.375,1.953)$ & 0.712 & 0.999 & $(0.427,2.340)$ & 0.999 \\
\hline \multirow[t]{3}{*}{ Tumor size (cm) } & & & & 0.003 & & & 0.002 \\
\hline & $<1 \mathrm{~cm}$ & 1 & & & 1 & & \\
\hline & $\geq 1 \mathrm{~cm}$ & 1.822 & $(1.222,2.717)$ & 0.003 & 1.959 & $(1.283,2.990)$ & 0.002 \\
\hline \multirow{3}{*}{ Extension } & $\begin{array}{l}\text { Major vascular invasion: majorbranch of portal } \\
\text { or hepatic vein(s) }\end{array}$ & 1.751 & $(0.591,5.185)$ & 0.312 & 1.573 & $(0.503,4.921)$ & 0.436 \\
\hline & Hepatic artery or vena cava invasion & 2.794 & $(0.569,13.726)$ & 0.206 & 4.238 & $(0.804,22.337)$ & 0.089 \\
\hline & $\begin{array}{l}\text { Coronary, falciform, hepatoduodenal, } \\
\text { hepatogastric, round (of liver), triangular, } \\
\text { perforation of visceral peritoneum, parietal } \\
\text { peritoneum, pancreas, pleura, stomach } \\
\text { extension }\end{array}$ & 0.561 & $(0.192,1.641)$ & 0.291 & 0.835 & $(0.260,2.678)$ & 0.761 \\
\hline
\end{tabular}

Table 3 (continued) 
Table 3 (continued)

\begin{tabular}{|c|c|c|c|c|c|c|c|}
\hline Parameter & Subgroup & \multicolumn{3}{|c|}{ OS } & \multicolumn{3}{|c|}{ DSS } \\
\hline \multirow[t]{7}{*}{ Surgery } & & & & 0.000 & & & 0.000 \\
\hline & None & 1 & & & 1 & & \\
\hline & Local tumor destruction & 0.505 & $(0.285,0.895)$ & 0.019 & 0.464 & $(0.242,0.890)$ & 0.021 \\
\hline & Lobectomy & 0.216 & $(0.118,0.394)$ & 0.000 & 0.244 & $(0.131,0.454)$ & 0.000 \\
\hline & Extended lobectomy & 0.349 & $(0.107,1.138)$ & 0.081 & 0.296 & $(0.070,1.243)$ & 0.096 \\
\hline & Hepatectomy & 0.070 & $(0.016,0.312)$ & 0.000 & 0.050 & $(0.006,0.386)$ & 0.004 \\
\hline & Total hepatectomy + transplant & 0.074 & $(0.025,0.219)$ & 0.000 & 0.065 & $(0.018,0.237)$ & 0.000 \\
\hline \multirow{4}{*}{ AJCC stage } & I & 1 & & & 1 & & \\
\hline & II & 2.305 & $(0.595,8.923)$ & 0.227 & 1.639 & $(0.382,7.038)$ & 0.506 \\
\hline & III & 3.143 & $(0.978,10.101)$ & 0.055 & 2.598 & $(0.709,9.520)$ & 0.150 \\
\hline & IV & 0.999 & $(0.141,7.063)$ & 0.999 & 0.774 & $(0.100,5.972)$ & 0.806 \\
\hline
\end{tabular}

Table 3: tumor size $(\mathrm{cm})$ and surgery were the independent prognostic factors of the OS and DSS of PCCCL patients. 0.000 means $\mathrm{P}<0.001$. DSS, disease-specific survival; OS, overall survival; HR, hazard ratio; $\mathrm{Cl}$, confidence interval; AJCC, American Joint Committee on Cancer; PCCCL, primary clear cell carcinoma of liver.

Table 4 Final model

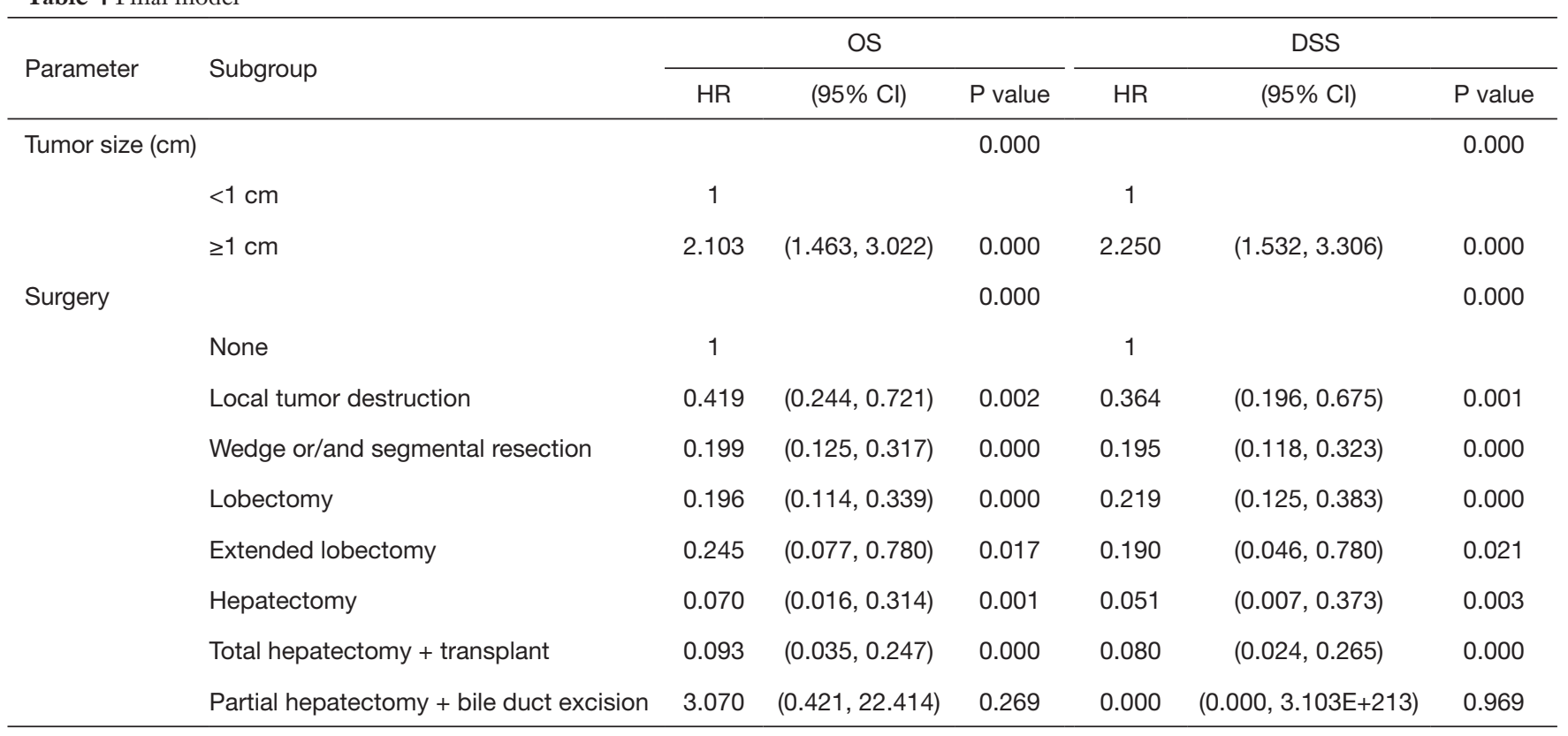

Table 4: tumor size (cm) and surgery were the independent prognostic factors of the OS and DSS of PCCCL patients. 0.000 means $\mathrm{P}<0.001$. OS, overall survival; DSS, disease-specific survival; $\mathrm{HR}$, hazard ratio; $\mathrm{Cl}$, confidence interval; PCCCL, primary clear cell carcinoma of liver. 
Table 5 Multivariate analysis of clinical and pathological prognostic factors in PCCCL by using competing risk model

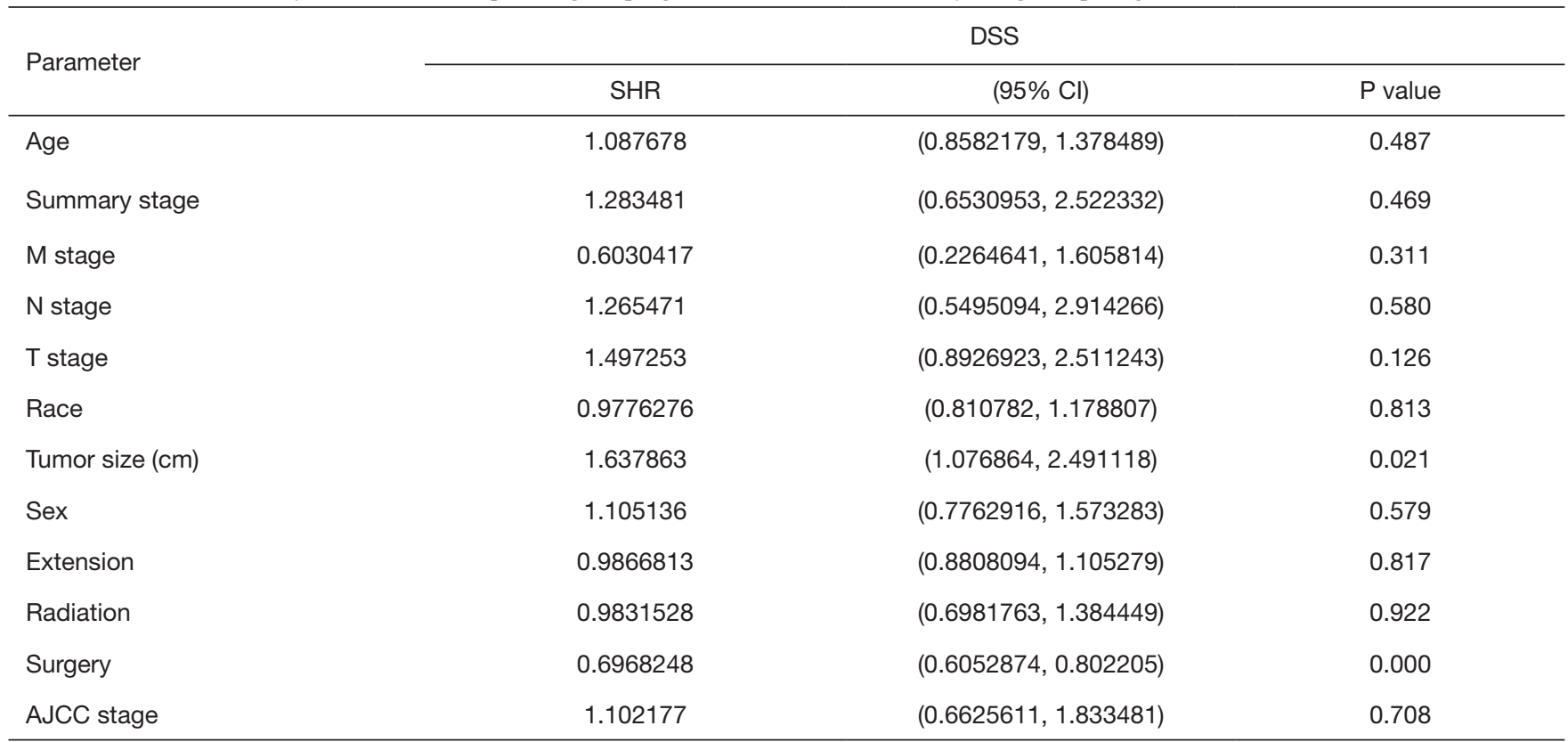

Table 5: regression results of competitive risk model for PCCCL patients. 0.000 means $\mathrm{P}<0.001$. PCCCL, primary clear cell carcinoma of liver; DSS, disease-specific survival; SHR, subdistribution HR; HR, hazard ratio; CI, confidence interval; AJCC, American Joint Committee on Cancer.
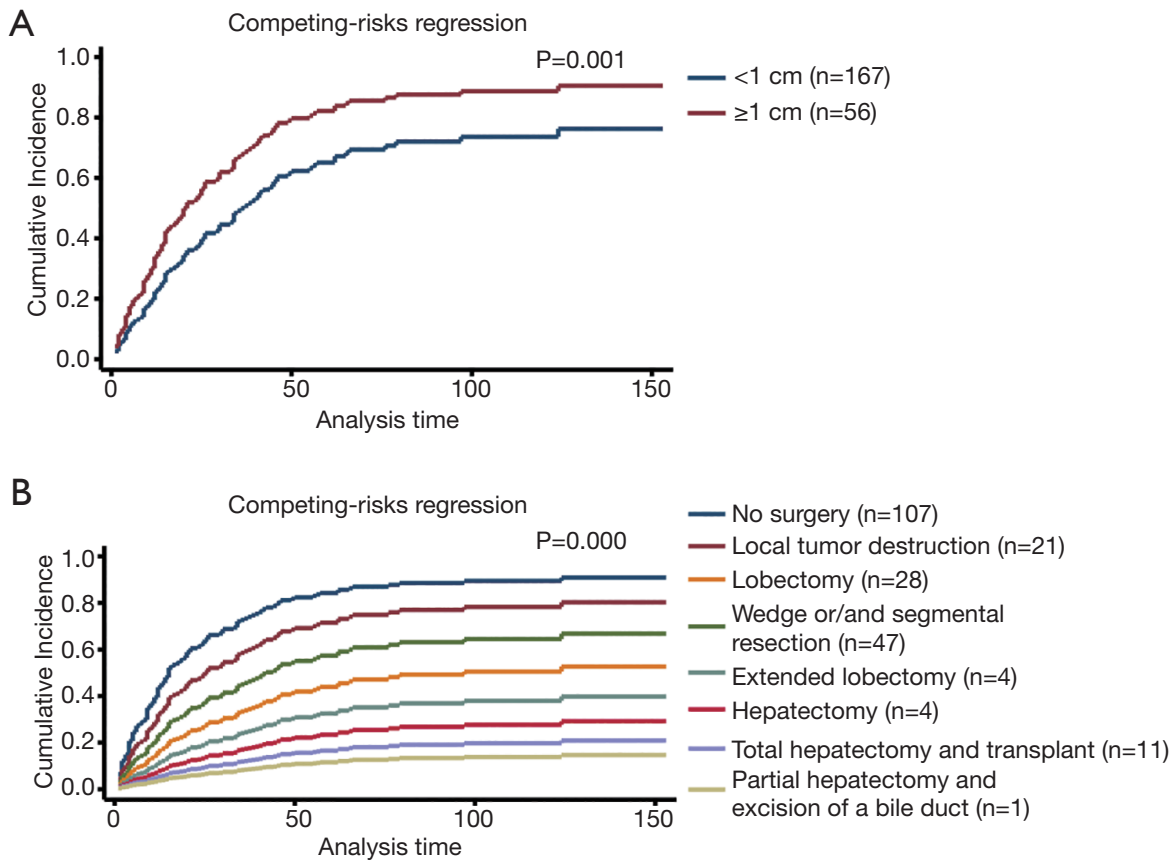

Figure $7 \mathrm{CIF}$ of interesting events in PCCCL according to different clinical and pathological factors in presence of competing risks was analyzed by Gray test. (A) Different tumor size at diagnosis; (B) different surgery method. 0.000 means $\mathrm{P}<0.001$. CIF, cumulative incidence function; PCCCL, primary clear cell carcinoma of liver. 
staining is a distinguishing feature of PCCCL (19). Owing to the relative rareness, most studies regarding PCCCL have been case reports or small, single-institution studies, rendering the epidemiology, etiology, and pathogenesis poorly understood (20). To the best of our knowledge, the present study included the largest published cohort of PCCCL patients and was the first to characterize the clinicopathologic properties, demographic features, treatment outcomes, and prognostic factors.

PCCCL has previously been reported to have low mortality and incidence ( $<10 \%$ of HCC cases) $(12,13,21-23)$. A morbidity of $8.7 \%$ has been observed in America (22). Additionally, PCCCL has been detected in $9.3 \%$ (9), and $6.7-13.3 \%(4,24)$ of the HCC patients in Japan and China, respectively. As expected, our results confirmed the rarity of PCCCL; we found that, between 2004 and 2016, 33,345 cases (data not shown) were diagnosed as single primary HCC with positive histology confirmation, and 349 of them (1.05\%) were PCCCL.

In this study, the average age of PCCCL patients was 63.8 (range: $18-94$ ) years, which was older than those reported by Liu (14) (52 years) and Lee (25) (58.8 years). Regarding sex, our study indicated a male-to-female ratio of 1.34 , indicating that males were more prone than females. This observation is in agreement with previous reports $(4,6)$. Additionally, most patients (63.2\%) were of white ethnicity, reflecting the race distribution of western population. We also observed that the tumors were mostly $(74.9 \%)<1 \mathrm{~cm}$. It is extremely rare for HCC to be found in less than $1 \mathrm{~cm}$ in size. And it may be attributable to that PCCCL tend to have tumor capsule formation. Our observation differed from the previous observations of the high prevalence of big tumors of PCCCL [ $>2.1 \mathrm{~cm}(9)$ or $7.28 \mathrm{~cm}$ (14) on average]. This difference was likely due to the relatively small numbers of cases in these two previous studies (20 cases per study). Furthermore, the observations of Kida et al. are in agreement with ours $(12,26)$.

In this study, more PCCCL patients were diagnosed as T1 (49.8\%), N0 (96.0\%), or M0 (87.4\%) stage, in addition to more AJCC stage I (44.4\%) or localized stage (63.7\%) patients, in accordance with several studies that have shown that PCCCL tends to have less vascular invasion $(4,12)$, lymph node metastases (14), and extra- or intrahepatic metastasis (3). This may be attributable to the high incidence of tumor capsule formation $(11,27)$, presumably caused by the stable expression of type I and III procollagen $(4,28)$. In line with this, our study showed that the patients without vascular invasion accounted for $63.2 \%$ of the sample size.

The clinical prognosis of PCCCL patients remains disputed. Most studies have indicated that patients with PCCCL had better outcome than those with other subtypes of HCC $(10,22,29,30)$. Liu et al. (4) have reported that PCCCL patients had a 5-year OS rate of $39 \%$ and a median OS of 40 months. Xu et al. (5) have suggested a good prognosis, with a 5 -year OS and DSS of $58.5 \%$ of $48.6 \%$, respectively, whereas another study (11) has estimated 35.9\% and $28.1 \%$. Even the spontaneous regression of primary and metastatic PCCCL lesions occasionally occur (19). Meanwhile, several studies have claimed that the prognosis of PCCCL is poor (9). However, the limited numbers of cases render these conclusions unreliable. Here, we observed a 5 -year OS and DSS of $24.3 \%$ and $29.4 \%$, respectively, and a median OS and DSS of 21 and 26 months. Accordingly, our findings support the former view that PCCCL patients have a relatively bad clinical outcome. Although our results are inconsistent with the mainstream, we have included 223 cases of PCCCL patients in the cohort study, reinforcing the reliability of our conclusions.

Kaplan-Meier, Cox regression analyses and the regression results of multivariate competitive risk model revealed that the increased tumor size was independently correlated with low OS and DSS rates in PCCCL patients. This association agreed with the conclusion of Chen et al. (11) that tumor size was a prognostic indicator for OS, disagreeing with $\mathrm{Xu}$ et al. (5), who have claimed that the Edmondson grade is the only independent risk. This difference may be attributable to the involvement of a small patient cohort (38 cases in the study of $\mathrm{Xu}$ et al.), hindering an accurate estimation.

Surgery may be the most important factor affecting the survival. Since PCCCL is more prone to forming capsules, surgical resection is an effective way to eliminate lesions containing intact capsules, thereby improving the survival (31). Surgery has been suggested to provide a longterm survival for PCCCL patients (27). Here, we found that the PCCCL patients who received no surgery had a shorter survival (median OS: 9 months; median DSS: 10 months) than those who underwent surgeries. We observed that total hepatectomy and transplant combination offered the longest survival (median OS: 124 months) for the PCCCL patients, followed by hepatectomy (median OS and DSS: both 62 months), wedge or/and segmental resection (median OS: 56 months and median DSS: 62 months) and extended lobectomy (median OS and DSS: both 41 months). And the regression results of multivariate competitive risk model also showed the similar trend. However, we found that 
partial hepatectomy along with bile duct excision offered a poor survival (median OS: 5 months) but a least risk for DSS. It may be explained that there was only one patient in this group, which would lead to deviation and cannot represent the overall level. Therefore, surgical interventions should be used as the first-line treatment.

Although surgical resection is effective in treating PCCCL, adjuvant treatments, such as radiation therapy, has increasingly been receiving attention. Radiation therapy has been reported as an important clinical application in various tumors (32). However, our results showed that radiotherapy had no significant effect on the prognosis of PCCCL patients. This observation may be due to the special pathological type of PCCCL. Similarly, traditional chemo- and radio-therapy are largely ineffective in treating any renal cell carcinoma subtype, and the underlying mechanistic reasons need to be further explored (33).

Similar with other retrospective studies using data from the SEER database, there are some limitations in our research. Firstly, the detailed chemotherapy information was not available in the database. Lack of these data is not conducive to our understanding of the treatments for PCCCL. Secondly, the patient data mainly illustrated the clinical characteristics of PCCCL in America and might not be globally applicable. Thirdly, we failed to collect the data about the proportion of clear cells and capsule formation. However, most studies indicated that capsule formation or high proportion of clear cells is beneficial to prolong the survival time (5). Fourthly, there are no data about any PCCCL-related genetic abnormalities in the SEER database. As known, oncogenic mutations or DNA abnormalities play an important role in the progression and chemo- and radiosensitivities of various tumors (34). Aggressive morphologic features and aneuploidy have been reported to be associated with the prognosis of PCCCL (35). Clear cell HCC shows a higher frequency of IDH1 mutation, which is associated with shorter survival times (25). Lack of such information limits our full understanding of PCCCL. We should notice the limitation of patient size which should be overcome by collecting more cases for a longer time.

In conclusion, in our exploratory research on PCCCL patients, we used the SEER dataset to characterize the demographic, clinical, survival, and therapeutic features of PCCCL patients. We found that PCCCL was more common in white people and prone to be localized, early with small size and weak aggressiveness. Our study showed a relatively poorer outcome of PCCCL than previous studies. Moreover, tumor size and surgery were independent prognostic factors for the OS and DSS of the PCCCL patients. Surgery intervention could improve the outcome; however, radiotherapy failed to lengthen the survival time.

\section{Acknowledgments}

Funding: This work was supported by China Postdoctoral Science Funds of JW (2020M683115), the National Natural Science Foundation of China (No. 81902909 to AA) and the fund of Peking University People's Hospital (RDY201923 to $\mathrm{XY}$ ).

\section{Footnote}

Reporting Checklist: The authors have completed the STROBE reporting checklist. Available at https://dx.doi. org/10.21037/tcr-21-9

Conflicts of Interest: All authors have completed the ICMJE uniform disclosure form (available at https://dx.doi. org/10.21037/tcr-21-9). The authors have no conflicts of interest to declare.

Ethical Statement: The authors are accountable for all aspects of the work in ensuring that questions related to the accuracy or integrity of any part of the work are appropriately investigated and resolved. The study was conducted in accordance with the Declaration of Helsinki (as revised in 2013). As the patients data in the SEER dataset are publicly available, therefore, no approval was required from any institutional review board. Informed consent was waived.

Open Access Statement: This is an Open Access article distributed in accordance with the Creative Commons Attribution-NonCommercial-NoDerivs 4.0 International License (CC BY-NC-ND 4.0), which permits the noncommercial replication and distribution of the article with the strict proviso that no changes or edits are made and the original work is properly cited (including links to both the formal publication through the relevant DOI and the license). See: https://creativecommons.org/licenses/by-nc-nd/4.0/.

\section{References}

1. Luo X, Sui J, Yang W, et al. Type 2 diabetes prevention diet and hepatocellular carcinoma risk in US men and women. Am J Gastroenterol 2019;114:1870-7. 
2. Zakka K, Jiang R, Alese OB, et al. Clinical outcomes of rare hepatocellular carcinoma variants compared to pure hepatocellular carcinoma. J Hepatocell Carcinoma 2019;6:119-29.

3. Xiong J, He D, Hu W, et al. Retroperitoneal and intrahepatic metastasis from primary clear cell carcinoma of the liver: a case report and review of the literature. Medicine (Baltimore) 2017;96:e6452.

4. Liu Z, Ma W, Li H, et al. Clinicopathological and prognostic features of primary clear cell carcinoma of the liver. Hepatol Res 2008;38:291-9.

5. $\mathrm{Xu} W, \mathrm{Ge} \mathrm{P}, \mathrm{Liao} W$, et al. Edmondson grade predicts survival of patients with primary clear cell carcinoma of liver after curative resection: a retrospective study with long-term follow-up. Asia Pac J Clin Oncol 2017;13:e312-20.

6. Wang H, Tan B, Zhao B, et al. CT findings of primary clear cell carcinoma of liver: with analysis of 19 cases and review of the literature. Abdom Imaging 2014;39:736-43.

7. El Jabbour T, Lagana SM, Lee H. Update on hepatocellular carcinoma: pathologists' review. World J Gastroenterol 2019;25:1653-65.

8. Ji SP, Li Q, Dong H. Therapy and prognostic features of primary clear cell carcinoma of the liver. World J Gastroenterol 2010;16:764-9.

9. Yang SH, Watanabe J, Nakashima O, et al. Clinicopathologic study on clear cell hepatocellular carcinoma. Pathol Int 1996;46:503-9.

10. Emile JF, Lemoine A, Azoulay D, et al. Histological, genomic and clinical heterogeneity of clear cell hepatocellular carcinoma. Histopathology 2001;38:225-31.

11. Chen ZS, Zhu SL, Qi LN, et al. Long-term survival and prognosis for primary clear cell carcinoma of the liver after hepatectomy. Onco Targets Ther 2016;9:4129-35.

12. Kida K, Oida T, Mimatsu K, et al. Hand-assisted laparoscopic hepatectomy for primary clear cell hepatocellular carcinoma of the liver. Case Rep Gastroenterol 2012;6:328-32.

13. Clayton EF, Furth EE, Ziober A, et al. A case of primary clear cell hepatocellular carcinoma in a non-cirrhotic liver: an immunohistochemical and ultrastructural study. Rare Tumors 2012;4:e29.

14. Liu QY, Li HG, Gao M, et al. Primary clear cell carcinoma in the liver: CT and MRI findings. World J Gastroenterol 2011;17:946-52.

15. Takahashi A, Saito H, Kanno Y, et al. Case of clear-cell hepatocellular carcinoma that developed in the normal liver of a middle-aged woman. World J Gastroenterol
2008;14:129-31.

16. Daly MC, Paquette IM. Surveillance, Epidemiology, and End Results (SEER) and SEER-Medicare Databases: use in clinical research for improving colorectal cancer outcomes. Clin Colon Rectal Surg 2019;32:61-8.

17. Chen B, Liu B, Wu C, et al. Prognostic factors among single primary gliosarcoma cases: a study using Surveillance, Epidemiology, and End Results data from 1973-2013. Cancer Med 2019;8:6233-42.

18. Duggan MA, Anderson WF, Altekruse S, et al. The Surveillance, Epidemiology, and End Results (SEER) program and pathology: toward strengthening the critical relationship. Am J Surg Pathol 2016;40:e94-102.

19. Jeon SW, Lee MK, Lee YD, et al. Clear cell hepatocellular carcinoma with spontaneous regression of primary and metastatic lesions. Korean J Intern Med 2005;20:268-73.

20. Kokubo R, Saito K, Shirota N, et al. A case of primary clear cell hepatocellular carcinoma comprised mostly of clear cells. Radiol Case Rep 2019;14:1377-81.

21. Wu PC, Lai CL, Lam KC, et al. Clear cell carcinoma of liver. An ultrastructural study. Cancer 1983;52:504-7.

22. Buchanan TF, Jr., Huvos AG. Clear-cell carcinoma of the liver. A clinicopathologic study of 13 patients. Am J Clin Pathol 1974;61:529-39.

23. Fu LY, Mitchell KA, Cai G. Clear cell hepatocellular carcinoma diagnosed by bile duct brushing cytology. Diagn Cytopathol 2016;44:147-51.

24. Wang Y, Zhang J, Gao Y, et al. Therapeutic efficacy of transcatheter arterial embolization of primary hepatocellular carcinoma: discrepancy in different histopathologic subtypes. Chin Med J (Engl) 1999;112:264-8.

25. Lee JH, Shin DH, Park WY, et al. IDH1 R132C mutation is detected in clear cell hepatocellular carcinoma by pyrosequencing. World J Surg Oncol 2017;15:82.

26. Li T, Fan J, Qin LX, et al. Risk factors, prognosis, and management of early and late intrahepatic recurrence after resection of primary clear cell carcinoma of the liver. Ann Surg Oncol 2011;18:1955-63.

27. Lao XM, Zhang YQ, Jin X, et al. Primary clear cell carcinoma of liver--clinicopathologic features and surgical results of 18 cases. Hepatogastroenterology 2006;53:128-32.

28. Torimura T, Ueno T, Inuzuka S, et al. Mechanism of fibrous capsule formation surrounding hepatocellular carcinoma. Immunohistochemical study. Arch Pathol Lab Med 1991;115:365-71.

29. Intraobserver and interobserver variations in liver biopsy 
interpretation in patients with chronic hepatitis C. The

French METAVIR Cooperative Study Group. Hepatology 1994;20:15-20.

30. Salvucci M, Lemoine A, Azoulay D, et al. Frequent microsatellite instability in post hepatitis B viral cirrhosis. Oncogene 1996;13:2681-5.

31. Liu JH, Tsai HL, Hsu SM, et al. Clear cell and non-clear cell hepatocellular carcinoma: a case report and literature review. Kaohsiung J Med Sci 2004;20:78-82.

32. Sampath $S$. Treatment: radiation therapy. Cancer Treat Res 2016;170:105-18.

Cite this article as: Wen J, Yao X, Xue L, Aili A, Wang J. Predictors and survival of primary clear cell carcinoma of liver: a population-based study of an uncommon primary liver tumor. Transl Cancer Res 2021;10(7):3326-3344. doi: 10.21037/tcr-21-9
33. Makhov P, Joshi S, Ghatalia P, et al. Resistance to systemic therapies in clear cell renal cell carcinoma: mechanisms and management strategies. Mol Cancer Ther 2018;17:1355-64.

34. Pelosi A, Careccia S, Sagrestani G, et al. Dual promoter usage as regulatory mechanism of let-7c expression in leukemic and solid tumors. Mol Cancer Res 2014;12:878-89.

35. Orsatti G, Arnold MM, Paronetto F. DNA image cytometric analysis of primary clear cell carcinoma of the liver. Arch Pathol Lab Med 1994;118:1226-9. 